\title{
Active-Impedance Control of a Lower-Limb Assistive Exoskeleton
}

\author{
Gabriel Aguirre-Ollinger, J. Edward Colgate, Michael A. Peshkin and Ambarish Goswami
}

\begin{abstract}
We propose a novel control method for lower-limb assist that produces a virtual modification of the mechanical impedance of the human limbs. This effect is accomplished by making the exoskeleton display active impedance properties. Active impedance control emphasizes control of the exoskeleton's dynamics and regulation of the transfer of energy between the exoskeleton and the user. Its goal is improving the dynamic response of the human limbs without sacrificing the user's control authority. The proposed method is an alternative to myoelectrical exoskeleton control, which is based on estimating muscle torques from electromyographical (EMG) activity. Implementation of an EMG-based controller is a complex task that involves modeling the user's musculoskeletal system and requires recalibration. In contrast, active impedance control is less dependent on estimation of the user's attempted motion, thereby avoiding conflicts resulting from inaccurate estimation.

In this paper we also introduce a new form of human assist based on improving the kinematic response of the limbs. Reduction of average muscle torques is a common goal of research in human assist. However, less emphasis has been placed so far on improving the user's agility of motion. We aim to use active impedance control to attain such effects as increasing the user's average speed of motion, and improving their acceleration capabilities in order to compensate for perturbations from the environment.
\end{abstract}

Index Terms-Exoskeleton, lower limb, active impedance, assist.

\section{INTRODUCTION}

In recent years, different exoskeletal devices have been developed to supplement the mechanical power needed by people with lower-limb impairments. Control methods for power assist are usually based on obtaining an estimate of the user's intended motion in real time. Estimates of intent based on the muscles' electromyographical (EMG) activity or on ground-reaction forces have been implemented with varying levels of success.

In this paper we establish the basic framework for a novel control method for lower-limb assistive exoskeletons based on active impedance. This method gives preeminence to control of the exoskeleton's dynamics over prediction of intent. Our long-term goal is to develop a control method for exoskeletons that provides sufficient flexibility to assist a wide variety of lower-limb motions, such as those encountered in activities of daily living (ADL): walking, climbing stairs, rising from a chair, etc. Simultaneously,

This project has been supported through a grant from the Honda Research Institute (Mountain View, CA).

Gabriel Aguirre-Ollinger (gaguirreanorthwestern. edu), J. Edward Colgate and Michael A. Peshkin are with the Department of Mechanical Engineering, Northwestern University, Evanston, IL 60208, USA.

Ambarish Goswami is with the Honda Research Institute, Mountain View, CA 94041, USA. we introduce a new approach to human assist based on enhancing the kinematic response of the human limbs, as opposed to amplifying muscle forces. Because our method is not directly linked to a model of the user's biomechanics, we expect greater ease in adapting the controller to individual users. Our proposed control method may eventually find its application in machinery for the rehabilitation of the lower extremities, or in autonomous exoskeletal devices designed to assist in ADL.

\section{A CLASSIFICATION OF ASSISTIVE EXOSKELETONS: PASSIVE VS. ACTIVE}

An exoskeleton is a powered wearable device, typically with an anthropomorphic configuration, designed to track the user's motion, and interact with the user through force display. Exoskeleton designs can be classified in terms of their assistive capabilities as either passive or active devices. A passive device is one that cannot deliver more energy to the environment than it has previously drawn from the environment. Exoskeletons that display passive behavior assist human users mainly by helping them employ their own muscle power more effectively, but do not actually supply energy to the user. Examples of exoskeleton-based passive assist include:

- Passive gravity support: unactuated orthotic devices have been available for decades. In many instances they provide partial support of the user's weight by providing a mechanical path to the ground. A more elaborate gravity-support device based entirely on passive mechanical elements has been reported by Banala [2].

- Load-carrying assist: a special case of gravity support, this type of assist is typified by the BLEEX system developed at UC Berkeley [11], in which the exoskeleton partially or totally supports a load carried by the user, for example a heavy backpack. This device employs a novel control scheme that, by means of positive kinematic feedback, enhances the device's mechanical admittance, thus enabling it to closely track the human's movements. However, the device is still passive from the perspective of its interaction with the human user.

- Force-offsetting assist: as a complement to loadcarrying capabilities, the system developed by Walsh et al. [21] uses passive devices like springs to store energy released during negative-work phases of the gait cycle and releases it during the positive-work phases to assist the progression of the walking motion.

- Resonance-based assist: the exoskeleton modifies the dynamics of the limb to make it function closer to its 
resonant frequency, thus helping to make more effective use of the user's own muscle power [20].

Active devices, on the other hand, behave as energy sources. In order to be able to assist the widest possible variety of lower-limb motions, an exoskeleton must be capable of active behavior. One of the reasons is that human movements involve the elevation of the center of mass of the body (CoM) at one point or another. Only an active device can assist such motion in a repetitive way. As for walking on level ground, Donelan et al. [12] have demonstrated that step-to-step transitions, to the degree they involve redirecting the trajectory of the CoM, involve a net amount of work and, in consequence, an energetic cost to the human.

Probably the most common approach to active assist is using the muscles' electromyographical (EMG) activity to control the actuators of the exoskeleton or orthosis. Rosen et al. [19] have developed a powered exoskeleton to assist elbow motion, using EMG as the primary command signal. Ferris [7] and his group at the University of Michigan have designed a pneumatically powered, myoelectrically controlled ankle-foot orthosis as a tool for rehabilitation and study of human locomotor adaptation. One of the most advanced systems for lower-limb assist is the wearable robot HAL (Hybrid Assistive Limb) developed by Sankai [10] and his team at the University of Tsukuba. It includes four actuators for hip and knee assist, and uses feedback from both EMG and force sensors. Fleischer et al. [8] have developed a method for EMG-based torque estimation that tackles the important problem of calibrating the parameters of the torque estimator, through the use of a model of the human body's dynamics. This method has been used to implement a torque controller for a powered knee orthosis [9].

The use of EMG signals to estimate motion intent has several practical limitations, due mainly to the nature of the EMG signal. Accurate estimation of torque from EMG is a challenging task requiring the characterization of several muscles, plus separating extraneous components affecting the EMG signal, such as electrical noise, ambient noise and motion artifact. Muscle activation models derived from the Hill model are typically complex [15]. Furthermore, EMG displays considerable variability with time and across subjects, thus requiring recalibration schemes like the one described in [8]. A myoelectrical controller needs to be finetuned to each individual user, making adaptation to new users a complex task.

\section{Assist By ACTIVE IMPEDANCE: 1-DOF CASE}

\section{A. Passive and active mechanical impedance}

Our current research focuses on developing control methods to assist single-joint movements, as a preamble for the development of a control scheme for an exoskeleton with multiple degrees of freedom. The key aspect of our control method is enabling the exoskeleton to display active impedance properties.

Figure 1 shows a diagram of a hypothetical 1-DOF exoskeleton designed to assist the motion of the knee joint.
We shall model this device as an LTI second-order rotational system.

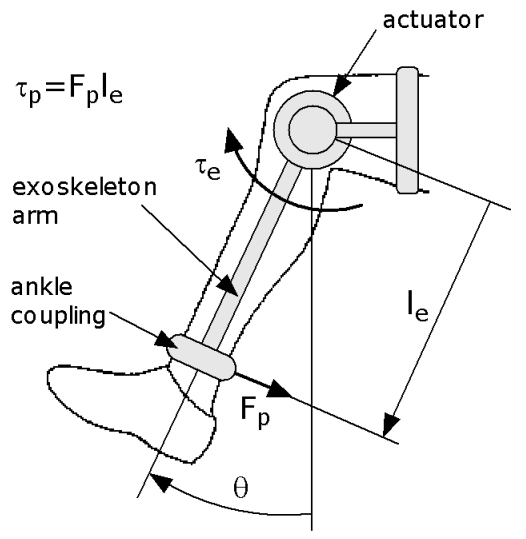

Fig. 1. Hypothetical 1-DOF exoskeleton.

Impedance control will be used to regulate the interaction between the exoskeleton and the user. Under this control regime, the exoskeleton will display a virtual mechanical impedance of our choice. The exoskeleton's virtual impedance parameters consist of inertia moment $I_{e}^{d}$, damping $b_{e}^{d}$, and stiffness $k_{e}^{d}$. The superscript $d$ denotes that these are "desired" impedance values. Thus we define the exoskeleton's virtual impedance as

$$
Z_{e}^{d}(s)=I_{e}^{d} s+b_{e}^{d}+\frac{k_{e}^{d}}{s}
$$

For the exoskeleton to be truly assistive, it must be capable of making the transition from passive to active behavior. A physical system is said to be passive if the amount of energy $\Delta E_{\text {out }}$ that can be extracted from it over a certain period of time $\Delta t$ is never greater than the sum of the system's initial energy $E_{o}$, plus the amount of energy $\Delta E_{i n}$ that entered the system during $\Delta t$ :

$$
\Delta E_{\text {out }} \leq E_{o}+\Delta E_{\text {in }}
$$

An active system, by contrast, is not subject to the above constraint. A port impedance $Z(s)$ is passive if and only if it meets the following conditions [4], otherwise it is active:

- $Z(s)$ has no poles in the right half of the complex plane.

- Any imaginary poles of $Z(s)$ are simple, and have positive residues.

- $\operatorname{Re}\{Z(j \omega)\} \geq 0$ for all $\omega$.

The relationship between passive and active impedance and energy transfer can be readily understood in terms of phase. Let us say the input velocity to the system described by (1) is sinusoidal, given by $v(t)=V \sin \omega t$. The steadystate output force will show a phase shift relative to $v(t)$ : $f(t)=F \sin (\omega t+\phi)$. The average power exchanged between the system and the environment is given by $P=\frac{F V}{2} \cos \phi$. A passive system is one in which the phase angle introduced by $Z(s)$ has a value between $-90^{\circ}$ and $90^{\circ}$; in this case the average power is positive, meaning the system draws energy from the environment. The system is active if the phase is 
either less than $-90^{\circ}$ or greater than $90^{\circ}$. In this case the average power is negative, meaning the system transmits energy to the environment. Because of the phase angle condition, the parameters of inertia, damping and stiffness in a passive system all have positive values. By contrast, an active system will display a certain combination of negative impedance terms. Negative masses, dampers or springs do not normally occur in nature. Thus an active impedance cannot be generated from ordinary mechanical elements, but requires an energy source plus control.

\section{B. Virtual modification of the human limb's impedance prop- erties}

We shall illustrate now how the exoskeleton can produce a virtual modification of the human leg's impedance. The impedance of the human leg's shank, $Z_{h}(s)$, is characterized by inertia moment $I_{h}$, damping $b_{h}$, and stiffness $k_{h}$. We are using the last term, $k_{h}$, mainly as a linearization of the gravitational torque acting on the leg, such that $m_{h} g l_{h} \sin \theta \approx$ $k_{h} \theta$.

$$
Z_{h}(s)=I_{h} s+b_{h}+\frac{k_{h}}{s}
$$

Figure 2 shows the coupled system formed by the exoskeleton's virtual impedance and the human limb. For simplicity, we assume the coupling between the exoskeleton and the human is rigid. The term $\tau_{p}$ represents the net torque exerted by the exoskeleton on the human limb at the interaction port $p ; \tau_{h}^{a}$ is the net muscle torque under exoskeleton assist.

It is easy to see that the equivalent impedance $Z_{h}^{d}(s)$ opposing the muscles' action is given by

$$
Z_{h}^{d}(s)=Z_{h}(s)+Z_{e}^{d}(s)
$$

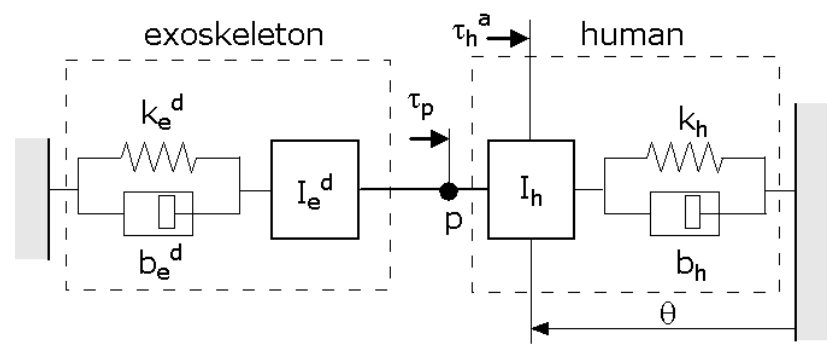

Fig. 2. Linear model of the coupled 1-DOF human-exoskeleton system.

Thus the dynamics of the coupled system are given by

$$
\left[\left(I_{h}+I_{e}^{d}\right) s+b_{h}+b_{e}^{d}+\left(k_{h}+k_{e}^{d}\right) \frac{1}{s}\right] \omega=\tau_{h}^{a}
$$

Equation (5) represents a generalized modification of the impedance parameters of the human limb segment by means of an exoskeleton. It suggests we have a wide range of possibilities to modify the relationship between the angular velocity trajectory, $\omega$, and the net muscle torque, $\tau_{h}^{a}$, by choosing the appropriate exoskeleton virtual impedance parameters $I_{e}^{d}, b_{e}^{d}$ and $k_{e}^{d}$.
We propose using the virtual modification of the lower limb's impedance as the primary source of human assist. Virtual impedance control of an exoskeleton has been proposed before [14] as a method of reducing the user's stress, but still giving preeminence to EMG-based torque amplification as the major assistive action.

Interaction between the actual human limb and the exoskeleton is of course more complex than that of the linear model described by (5). The passive impedance properties of the musculoskeletal system vary nonlinearly with the kinematic state of the body. On the other hand, people use impedance modulation (specifically by modifying joint stiffness through co-contraction) when interacting with unknown external forces [6]. In consequence, the actual implementation of active impedance control in an exoskeleton may eventually require making the virtual impedance terms dependent on the kinematic state of the limb or the interaction forces involved.

\section{EXPERIMENTAL PLATFORM: 1-DOF EXOSKELETON}

Our experimental platform is a 1-DOF exoskeleton mounted on a rigid base (Figure 3 ). Our current focus is being able to measure interaction forces and kinematic response with the least possible ambiguity. To that effect, it is ideal to start with a rigidly-mounted device that assists a simple motion of the leg. The exoskeleton is designed to assist a person performing knee extensions and flexions. A custombuilt ankle brace couples the user's leg to the exoskeleton arm. The ankle brace is mounted on a sliding bracket in order to accommodate any possible radial displacement of the ankle relative to the device's center of rotation.

The main consideration in the design of our experimental 1-DOF exoskeleton was enabling the display of programmed mechanical impedances, either active or passive, with high fidelity. We defer the design for actual use in rehabilitation or ADL for a later stage. For accurate impedance control it is ideal to employ a device with low intrinsic mechanical impedance, such that it is highly backdriveable even in the absence of motor actuation. Therefore, we chose to use a servo motor with a large torque capability, and keep the transmission ratio low.

Figure 4 shows the exoskeleton's main assembly, consisting of the servo motor, the drive transmission and the exoskeleton arm. The motor is a Kollmorgen brushless directdrive AC servo with a power rating of $0.99 \mathrm{~kW}$ and a continuous torque rating of $2.0 \mathrm{Nm}$. The transmission ratio of the cable drive is $10: 1$, thus allowing a continuous torque output of $20.0 \mathrm{Nm}$. The motor comes with a proprietary 24bit feedback device that produces an emulated encoder output of up to 32,768 counts before quadrature.

Design of the drive transmission is critical for activeimpedance assist. A gear transmission can be a poor choice for this type of application because gear backlash can give rise to limit cycles. Figure 5 shows a phase diagram of the behavior of a simulated 1-DOF exoskeleton with transmission backlash. 


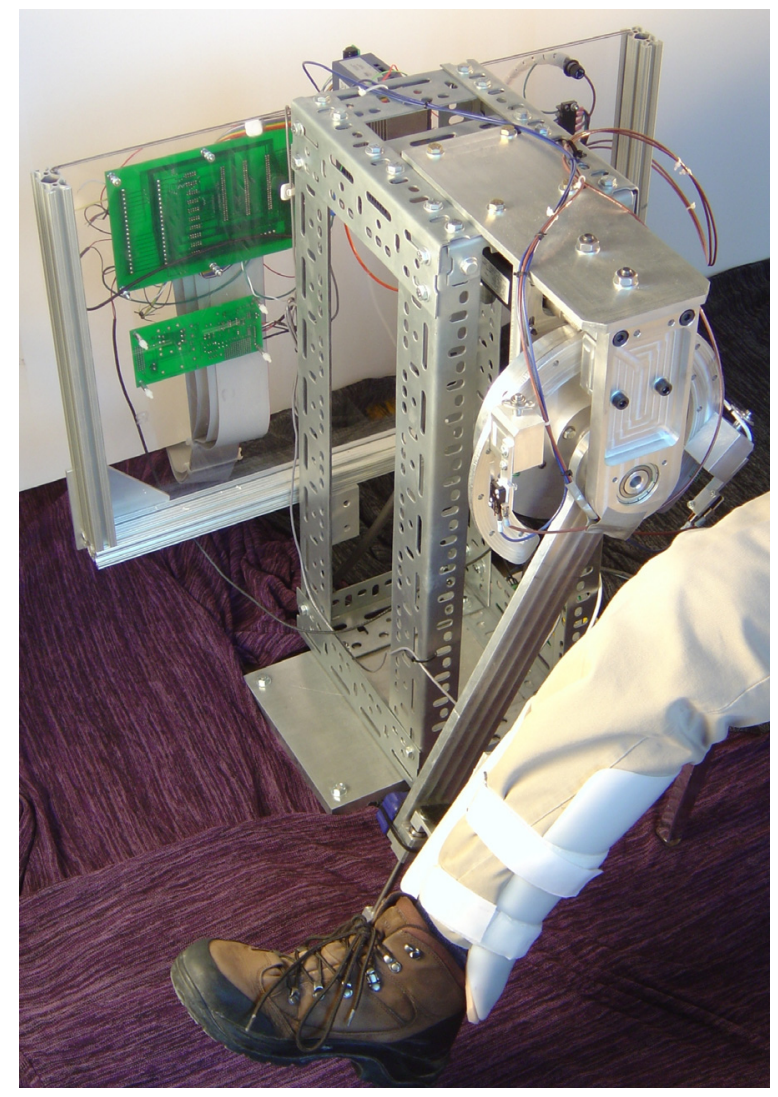

Fig. 3. 1-DOF exoskeleton for assisting knee-joint motion.

In this simulation, the exoskeleton's virtual impedance is a pure rotational inertia, $Z_{e}^{d}(s)=I_{e}^{d} s$. Coupling of the exoskeleton to a human leg at rest is represented by a spring and damper connected to ground. Noise in the torque measurement is simulated as a Gaussian white-noise signal.

In a backlash-free transmission, any motion induced by the torque sensor's signal noise will be attenuated by the damping in the coupling. In contrast, the presence of nonzero backlash predicts the onset of limit cycle, as shown in Figure 5. The amplitude of the limit cycle grows when negative virtual damping $b_{e}^{d}$ is introduced. In order to completely avoid the presence of backlash in our exoskeleton prototype, we decided to implement a cable drive transmission. The cable drive, shown also in Figure 4, is similar in concept to that of the PHANToM [13] and other haptic devices.

The interaction torque between the user's leg and the exoskeleton's arm is measured by means of a torque sensor coupling the arm to the major pulley of the drive. The arm's construction has been made as lightweight as possible in order to minimize its inertial effects. The torque sensor has a capacity of up to $113 \mathrm{Nm}$.

\section{IMPEDANCE CONTROLLER IMPLEMENTATION}

The general scheme for the control of the virtual impedance of our exoskeleton is shown in Figure 6. The torque $-\tau_{p}$ exerted by the exoskeleton on the human limb is used as input to a virtual admittance block, that is, a control block containing the reciprocal of the exoskeleton's desired

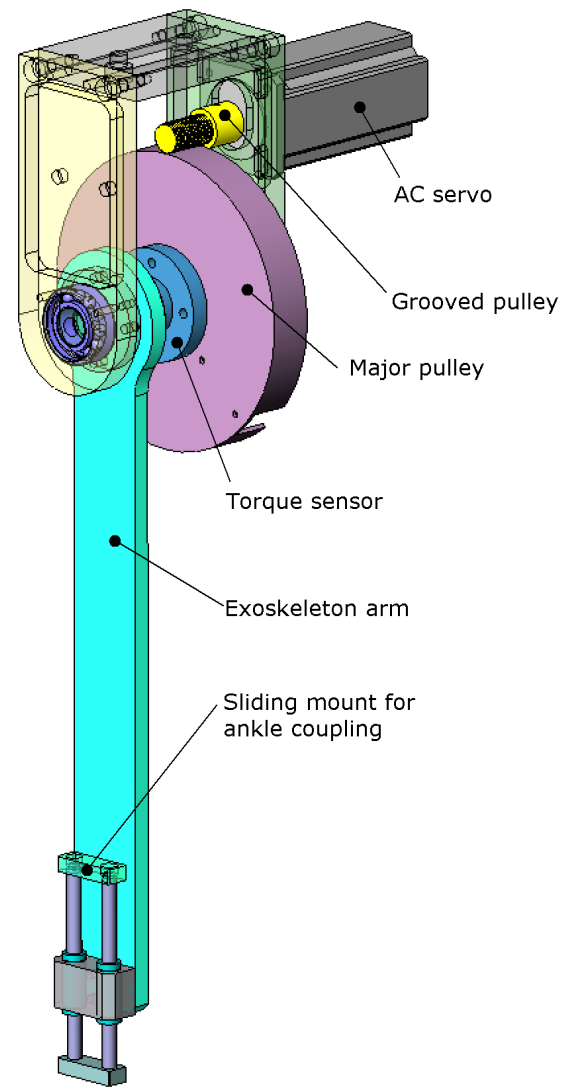

Fig. 4. Diagram of the 1-DOF exoskeleton's motor, drive and arm assembly.

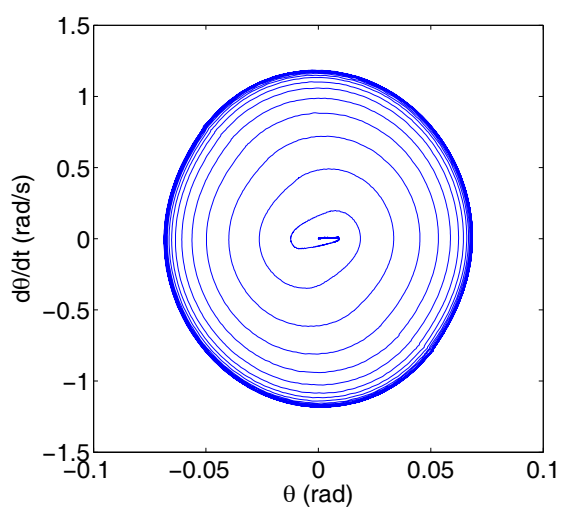

Fig. 5. Simulated limit cycle behavior in a 1-DOF exoskeleton with transmission backlash.

impedance $Z_{e}^{d}(s)$. The block in turn generates a reference velocity $\dot{\theta}_{\text {ref }}$. A complete kinematic reference trajectory $\mathbf{q}_{\text {ref }}$ (including the time integral of the angle to avoid steadystate error) is generated by a double integrator. This reference trajectory is tracked by a closed-loop PID controller using a state observer to compute an estimate of the actual kinematic trajectory, $\mathbf{q}_{\text {est }}$. The control code is implemented in the QNX real-time operating system, with a sampling rate of $1 \mathrm{kHz}$.

\section{ASSIST BY GRAVITY COMPENSATION}

While gravity compensation is not the primary focus of our interest, it is a useful benchmark to make an initial 


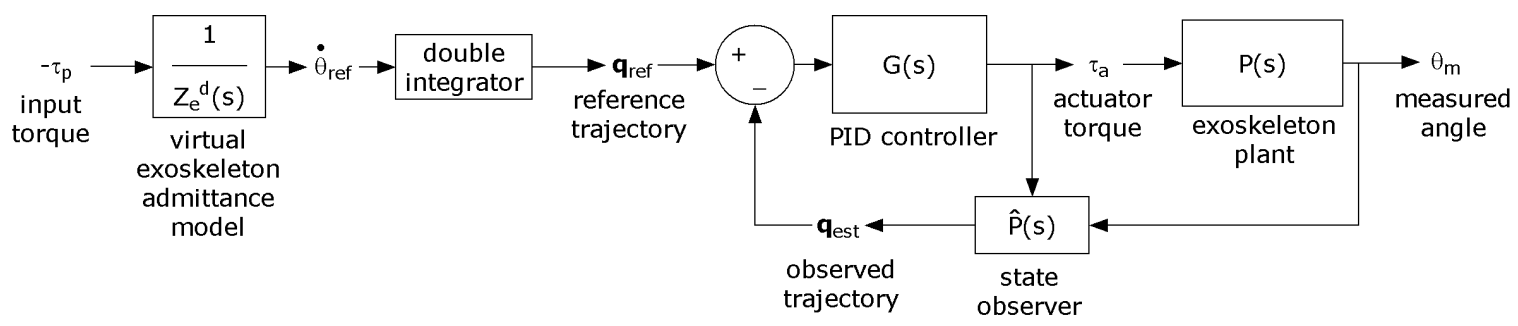

Fig. 6. Admittance controller for the 1-DOF exoskeleton.

assessment of the interaction between the human user and the exoskeleton. Because the term $k_{h}$ in our model of the human limb is used as a linearization of the effect of gravity, we can make the exoskeleton provide gravity compensation by giving a negative value to the virtual exoskeleton stiffness $k_{e}^{d}$, such that $k_{h}^{d}<k_{h}$.

The lead author served as the subject for the tests and performed knee extensions on the exoskeleton. During these exercises, we monitored the EMG activity of one muscle from the knee extensor group, the vastus lateralis. Processing of the raw EMG signal consisted of high-pass filtering at $30 \mathrm{~Hz}$ using a fourth-order Butterworth filter, full-wave rectification and low-pass filtering with a $6 \mathrm{~Hz}$ fourth-order Butterworth filter. Figure 7 shows plots of the EMG activity, $u_{E M G}$, and the knee joint angle, $\theta_{m}$, for two successive motions. The first motion was unassisted, i.e. the exoskeleton's virtual stiffness, $k_{e}^{d}$, had a value of zero. Prior to the second motion, $k_{e}^{d}$ was switched to a negative value of $-0.5 k_{h}$, effectively reducing the weight of the leg's shank by about $50 \%$. It can be seen that the gravity compensation controller achieved a significant reduction in the level of EMG activity.

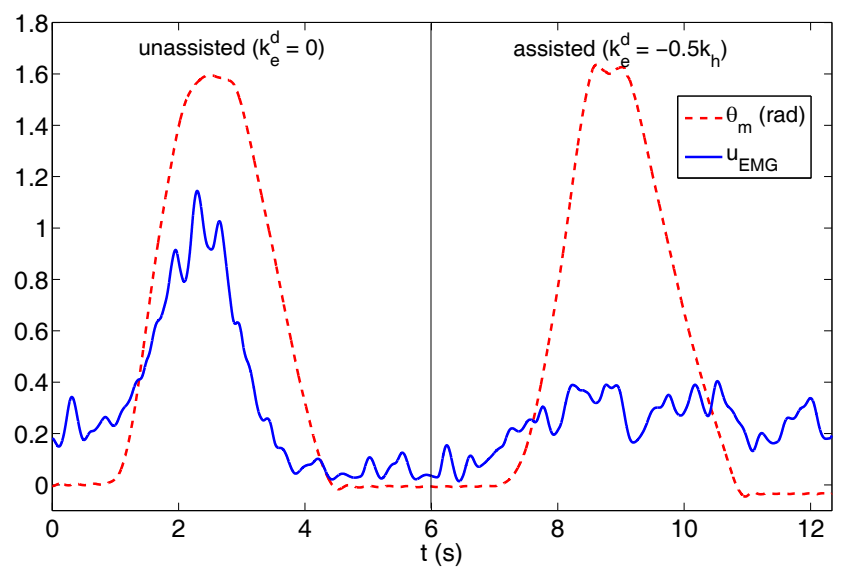

Fig. 7. Assist by gravity compensation: processed EMG levels for unassisted motion (first angle peak) and assisted motion (second angle peak).

\section{ASSIST BY IMPROVING THE HUMAN'S KINEMATIC RESPONSE}

The prevailing approach to lower-limb assist is reducing muscular effort. However, our research shows that little work has been done to study an exoskeleton's potential contribution to the agility or precision of lower-limb movements.
In this regard, trajectory-tracking tasks can be a useful tool to evaluate the effect of exoskeleton assist on the kinematic response of the lower limbs. Tracking tasks have been widely used in studies of the spatiotemporal control of the upper extremities. Studies on arm coordination involving targetreaching and trajectory-tracking under the action of external forces have been reported by Burdet [3], Dancause [5] and Patten [18]. As for studies on trajectory-tracking for the lower extremities, Maffiuletti [16] has reported on a lowerlimb trajectory-tracking task to assess motor coordination in able-bodied individuals.

We are currently developing new forms of human assist aimed at improving the kinematic response of the limb, for example by enabling the user to increase the magnitude of the velocity or the acceleration of their movements. Such effects may prove relevant to physical rehabilitation, inasmuch as they may help patients regain confidence in their lower-limb movements. In elderly people, the decline in sensorimotor control function leads to increased risk of falls and, consequently, a gradual loss of confidence during locomotion. Melzer et al. [17] have reported that the velocity of voluntary and compensatory stepping decreases dramatically with age. Age-related deterioration of physiological factors like sensory detection, cortex-level motion planning level and nerve conduction speed essentially cannot be helped. On the other hand, we feel that the exoskeleton can help to compensate the slower response of the nervous system by improving the dynamic response of the human limbs.

We are currently conducting a study to test the influence of negative damping, which is a particular case of active impedance, on the speed of leg movements executed in response to a sensory input. Because natural damping is an energy dissipation term, it makes sense to consider negative damping as a way to source energy from the exoskeleton to the user. A particular advantage of this method is that, since damping is a velocity-dependent effect, the exoskeleton-human interaction forces generated by the exoskeleton become nearly zero when the leg is at rest or in quasi-static motion. Our hypothesis is that negative exoskeleton damping can reduce the average time needed to complete the movement. The study involves target-reaching tasks adapted to the leg's motions. These experiments are designed in accordance with the stationary nature of our 1DOF exoskeleton platform. However, we expect the results will provide insights about the potential influence of an exoskeleton on the compensatory movements needed to 
maintain balance and respond to motion perturbations.

\section{KINEMATIC RESPONSE EXPERIMENTS WITH NEGATIVE DAMPING}

In our experiment, the subject moves the leg's shank while wearing the exoskeleton. The exoskeleton's measured angular position is used to guide a virtual object on a computer screen toward a fixed target, as shown in Figure 8(a). The visual interface is part of an integrated QNX application that runs the exoskeleton controller and collects the experimental data. The virtual object on the screen replicates the trajectory of the foot (Figure 8(b)), so that the center of its trajectory corresponds to the center of rotation of the knee. The virtual target is located on the path of the virtual object's trajectory. This reaching motion is repeated several times at different values of negative damping. The subject is instructed to move the virtual object to the target as quickly as possible, and hold it within the target's limits for a specific amount of time, typically a fraction of a second.

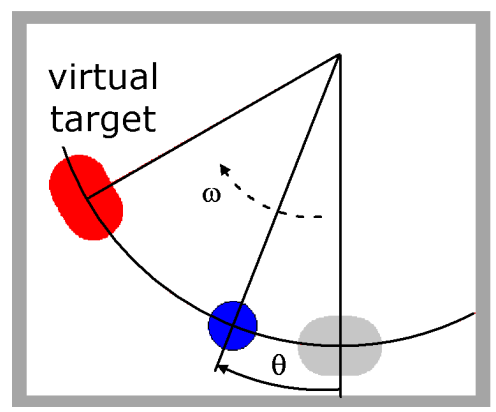

(a)

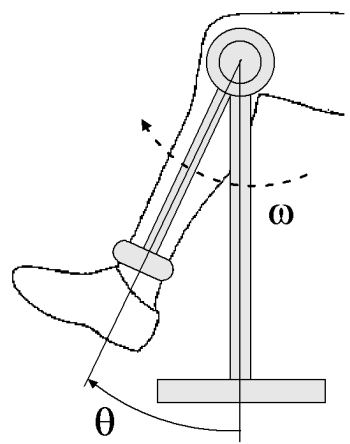

(b)
Fig. 8. Target-reaching task: (a) schematic diagram of the visual interface; (b) motion of the leg coupled to the exoskeleton.

\section{A. Identification of the dynamics of the exoskeleton and the lower limb}

We performed an initial test with the subject (healthy male, age 38 , height $1.82 \mathrm{~m}$, body mass $78 \mathrm{~kg}$ ) performing targetreaching motions at different levels of virtual exoskeleton damping. In order to assign appropriate values to the exoskeleton's damping, we identified the natural impedance parameters of the subject's lower leg. The systems identification procedure was done in two steps. First, the natural impedance parameters of the exoskeleton's arm were estimated using a recursive least-squares algorithm [1]. The system was excited with a band-limited $(3 \mathrm{~Hz})$ white-noise torque signal applied to the AC servo. In the recursive algorithm, the input variable was the measured torque, $\tau_{p}$, and the outputs were the angular position, $\theta_{e}$, angular velocity, $\dot{\theta}_{e}$, and angular acceleration, $\ddot{\theta}_{e}$, of the exoskeleton's arm. The angular acceleration was measured by means of an Xsens MT9 inertial unit. The estimated values for the inertia, damping and gravitational spring constant of the exoskeleton's arm were, respectively, $\hat{I}_{e}=0.116 \mathrm{kgm}^{2}, \hat{b}_{e}=0.058 \mathrm{Nm} /(\mathrm{rad} / \mathrm{s})$ and $\hat{k}_{e}=2.768 \mathrm{Nm} / \mathrm{rad}$.
The identification procedure was then repeated with the user's lower-leg coupled to the exoskeleton's arm. The subject was instructed to relax the leg as much as possible to insure that the identified parameters would reflect the passive dynamics of the leg. Assuming a fairly rigid coupling between the leg and the exoskeleton, the impedance parameters of the user's leg were estimated as $\hat{I}_{h}=0.220 \mathrm{kgm}^{2}$, $\hat{b}_{h}=1.714 \mathrm{Nm} /(\mathrm{rad} / \mathrm{s})$ and $\hat{k}_{h}=11.843 \mathrm{Nm} / \mathrm{rad}$. These values are in good agrement with estimates obtained from the anthropometric data tables in Winter [22].

The effect of negative exoskeleton damping $b_{e}^{d}$ can be viewed as a cancelation of the passive damping of the knee joint. Although the identified damping value $\hat{b}_{h}$ imposes a theoretical limit of $b_{e}^{d}=-1.714 \mathrm{Nm} /(\mathrm{rad} / \mathrm{s})$ to maintain stability, we found that in practice the subject was able to to perform the target-reaching task with values of exoskeleton damping as low as $-2.4 \mathrm{Nm} /(\mathrm{rad} / \mathrm{s})$ without loss of motor control. Thus we hypothesize that subjects may be quick to recognize the presence of excess negative damping, and to respond by increasing the joint's damping through either cocontraction or velocity feedback.

\section{B. Target-reaching experiment}

The virtual target was located at an angle of $60^{\circ}$ from the vertical (Figure 8). The subject performed the reaching motion at negative damping values $b_{e}^{d}$ of increasing magnitude. Based on the estimated passive damping, six uniformly distributed values of negative damping ranging from 0 to $-2.0 \mathrm{Nm} /(\mathrm{rad} / \mathrm{s})$ were used; six target-reaching motions were recorded for each value of damping. Three complete tests were performed in the study, giving a total of 108 targetreaching motions executed by the subject. The time to reach the target was recorded in every trial.

In computing the time to reach the target, we discounted the subject's reaction time, that is, the delay between the appearance of the visual cue (target) and the onset of leg movement. This delay is outside the influence of the exoskeleton and hence should not be counted. In order to have a fixed reference for the onset of the target-reaching motion, time computation was initiated when the leg crossed a threshold angle of $2.5^{\circ}$ measured $\mathrm{CW}$ from the vertical. As a measure of comfort for the user, the exoskeleton damping was returned to the default value of zero after the target was reached; in this way it is easier to move the leg back to the home position.

ANOVA performed on the experimental data showed that both the virtual damping, $b_{d}^{e}$, and the test number, $n_{t}$, had a highly significant effect on the time to reach the target. Figure 9 shows, for each test, a plot of the average time to reach the target, $t_{T A R G}$, versus the exoskeleton's virtual damping value, $b_{d}^{e}$. The plots show a consistent reduction in the time to reach the target as the virtual damping becomes more negative ( $P \simeq 0, R^{2}=0.647$ ). Reduction in the reaching time was also observed from test to test $\left(P \simeq 0, R^{2}=0.209\right.$ ). This suggests a possible learning effect, by which the user would be able to increase his average speed of motion as he becomes accustomed to wearing the exoskeleton. 


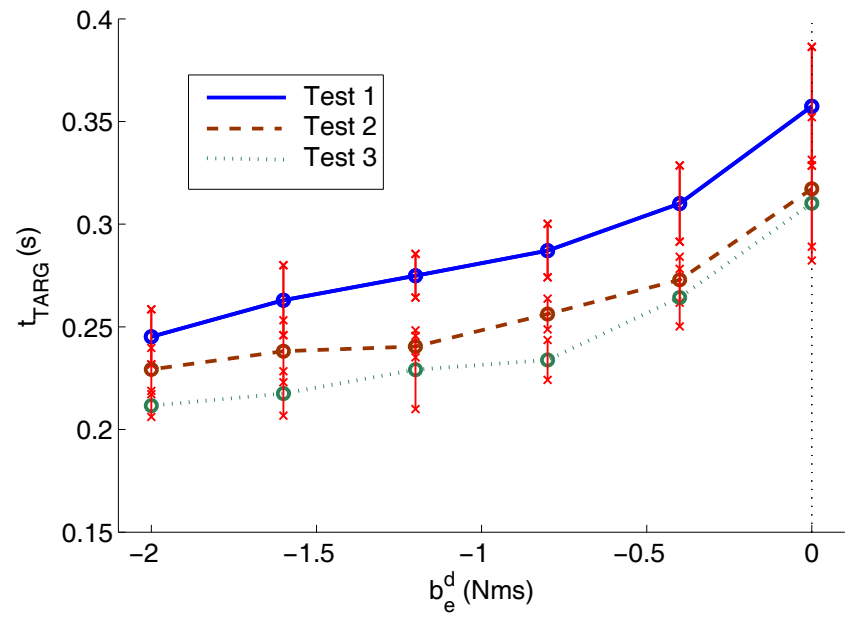

Fig. 9. Plots of average time to reach the target versus exoskeleton virtual damping.

\section{DISCUSSION}

The results from our initial experiment with negative exoskeleton damping, although limited in scope, support our hypothesis that negative damping contributes to reduce the time needed to complete a lower-limb motion. More detailed studies, involving several subjects, are being prepared to provide a complete assessment of the effects of active impedance and learning on the time to perform leg motions with the exoskeleton. We are also planning to study the effect of scaling the mass properties of the leg, through a combination of negative exoskeleton inertia and negative exoskeleton stiffness.

One of the main effects we expect to obtain from activeimpedance assist is increased the motion bandwidth of the leg during contact-free movements. In the case of a wearable exoskeleton, it is conceivable that a larger bandwidth would enable the user to impart greater accelerations to the leg and, as a consequence, improve his/her capability to perform corrective movements to maintain balance or avert a fall altogether.

We are also currently developing experiments to determine the effect of negative damping on the bandwidth of motions performed with the 1-DOF exoskeleton. One approach we are considering is modifying the target-reaching task so that a series of targets is presented to the subject at randomized time intervals and randomized positions. Spectral analysis of the target-reaching trajectories can be performed to determine the frequency content of assisted motions. An alternative approach would involve a trajectory-tracking task in which the subject is instructed to track a randomized angular trajectory with a certain frequency content. The subject's frequency-response function for this task can be identified and the effect of exoskeleton assist on the motion bandwidth can be assessed.

\section{Conclusions}

We have concluded a first stage in the development of a new method for exoskeleton-based lower-limb assist. The proposed method follows two main principles:

- Controlling the dynamics of the exoskeleton to make it display active impedance.

- Using the exoskeleton's assistive forces to improve the kinematic response of the user's limbs.

A major part of our initial work focused on implementing a 1-DOF platform to test the assistive effect of active impedance. The choice of a drive mechanism was found to be a critical factor in enabling the exoskeleton to display active impedances with fidelity. The implementation of the 1-DOF exoskeleton was validated with a test involving a conventional method of assist, namely gravity compensation of an open-loop lower-limb motion.

The second experiment, target-reaching under the action of negative exoskeleton damping, constituted an initial test of our kinematic-assist concept. Experimental results collected so far tend to support our hypothesis that negative damping contributes to reduce the time needed to complete a lower-limb motion. Future experimental work shall address the effect of negative damping and other forms of active impedance on the motion bandwidth of subjects using the exoskeleton.

\section{REFERENCES}

[1] K.J. Åström and B. Wittenmark. Adaptive Control. Prentice Hall, 2 edition, 1994

[2] S.K. Banala, S. K. Agrawal, A. Fattah, V. Krishnamoorthy, W.-L. Hsu, J. Scholz, and K. Rudolph. Gravity-balancing leg orthosis and its performance evaluation. IEEE Transactions on Robotics, 22(6):12281239, 2006.

[3] E. Burdet, K.P. Tee, I. Mareels, T.E. Milner, C.M. Chew, D.W. Franklin, R. Osu, and M. Kawato. Stability and motor adaptation in human arm movements. Biological Cybernetics, 94:20-32, 2006.

[4] E. Colgate and N. Hogan. The interaction of robots with passive environments: Application to force feedback control. Fourth International Conference on Advanced Robotics, 1989.

[5] N. Dancause, A. Ptito, and M.F. Levin. Error correction strategies for motor behavior after unilateral brain damage: short-term motor learning processes. Neuropsychologia, 40(8):1313-1323, 2002.

[6] P.R. Davidson and D.M. Wolpert. Motor learning and prediction in a variable environment. Current Opinion in Neurobiology, 13(2):232237, 2003.

[7] D.P. Ferris, J.M. Czerniecki, and B. Hannaford. An ankle-foot orthosis powered by artificial pneumatic muscles. Journal of Applied Biomechanics, 21:189-197, 2005.

[8] C. Fleischer, C. Reinicke, and G. Hommel. Predicting the intended motion with EMG signals for an exoskeleton orthosis controller. IEEE/RSJ International Conference on Intelligent Robots and Systems, pages 2029-2034, 2005.

[9] C. Fleischer, C. Reinicke, and G. Hommel. Torque control of an exoskeletal knee with EMG signals. Proceedings of the Joint Conference on Robotics: ISR 2006 and Robotik 2006, 2006.

[10] H. Kawamoto, Suwoong Lee, S. Kanbe, and Y. Sankai. Power assist method for HAL-3 using EMG-based feedback controller. IEEE International Conference on Systems, Man and Cybernetics, 2:1648$1653,2003$.

[11] H. Kazerooni, J.-L. Racine, L. Huang, and R. Steger. On the control of the Berkeley Lower Extremity Exoskeleton (BLEEX). IEEE International Conference on Robotics and Automation, pages 43534360, April 2005.

[12] A. Kuo, J.M. Donelan, and A. Ruina. Energetic consequences of walking like an inverted pendulum: Step-to-step transitions. Exercise and Sport Sciences Reviews, 33(2), 2005. 
[13] H.Y.K. Lau and L.C.C. Wai. Implementation of position force and position position teleoperator controllers with cable driven mechanisms. Robotics and Computer-Integrated Manufacturing, 21(2):145$152,2005$.

[14] S. Lee and Y. Sankai. Virtual impedance adjustment in unconstrained motion for an exoskeletal robot assisting the lower limb. Advanced Robotics, 19(7):773-795, 2005.

[15] D.G. Lloyd and T.F. Besier. An EMG-driven musculoskeletal model to estimate muscle forces and knee joint moments in vivo. Journal of Biomechanics, 36(6), 2003.

[16] N.A. Maffiuletti, M. Bizzini, S. Schatt, and U. Munzinger. A multijoint lower-limb tracking trajectory test for assessment of motor coordination. Neuroscience Letters, 384:106-111, 2005.

[17] I. Melzer and L.I.E. Oddsson. The effect of a cognitive task on voluntary step execution in healthy elderly and young individuals. Journal of the American Geriatrics Society, 52:1255-1262, 2004.

[18] C. Patten, J. Whitney, D. Kothari, J. Lexell, and P.S. Lum. Reliability and responsiveness of elbow trajectory tracking in chronic poststroke hemiparesis. Journal of Rehabilitation Research and Development, 40(6):487-500, 2003.

[19] J. Rosen, M. Brand, M.B. Fuchs, and M. Arcan. A myosignal-based powered exoskeleton system. IEEE Transactions on Systems, Man and Cybernetics, 31(3):210-222, 2001.

[20] M. Uemura, K. Kanaoka, and S. Kawamura. Power assist systems based on resonance of passive elements. IEEE International Conference on Intelligent Robots and Systems, pages 4316-4321, 2006.

[21] C.J. Walsh, D. Paluska, K. Pasch, W. Grand, A. Valiente, and H. Herr. Development of a lightweight, underactuated exoskeleton for loadcarrying augmentation. Proceedings of the 2006 IEEE International Conference on Robotics and Automation, pages 3485-3491, 2006.

[22] D.A. Winter. Biomechanics and Control of Human Movement. John Wiley and Sons, Inc., 3 edition, 2005. 